Geuther: Ueb. d. Einwirk. d. Phosphorchloride etc. 359

\begin{tabular}{rr}
\multicolumn{2}{c}{ Berechnet } \\
$\mathrm{B}=$ & 6,01 \\
$\mathrm{Cl}$ & $=77,60$ \\
$\mathrm{~N}$ & $=7,65$ \\
$\mathrm{O}$ & $=\frac{8,74}{100,00}$
\end{tabular}

Gefunden:

Die Trennung der Verbindung in zwei Schichten beirn Schmelzen rührt offenbar von einer theilweisen Zersetzung in $\mathrm{BCl}_{3}$ und in NOCl her. Die obere Schicht, welche ihre gelbe Farbe etwas darin gelöster Verbindung verdankt, besteht uffenbar aus Borchlorid, während die untere von geschmolzener Vcrbinding, der das Nitrosylchlorid beigemengt ist, gebildet wird.

Da der nach dem Abdestilliren der Verbindung im Cylinder verbleibende weisse Rückstand sich als Borsäureanhydrid erwies, so ist es wahrscheinlich, dass die Reaction der Hauptsache nach gemäss den Gleichungen:

$$
\begin{aligned}
& 2 \mathrm{BCl}_{3}+3 \mathrm{~N}_{2} \mathrm{O}_{4}=\mathrm{B}_{2} \mathrm{O}_{3}+6 \mathrm{NOCl}+3 \mathrm{O} \\
& \text { d. i. } \quad-\frac{6 \mathrm{BCl}_{3}+3 \mathrm{~N}_{2} \mathrm{O}_{4}}{=\mathrm{B}_{2} \mathrm{O}_{3}+6\left(\mathrm{BCl}_{3}, \mathrm{NOCl}\right)+3 \mathrm{O}}
\end{aligned}
$$

verlaufen ist.

\title{
Ueber die Einwirkung der Phosphorchloride auf die Phosphorsäuren;
}

$$
\text { ron }
$$

\section{Demselben.}

I. Trihydroxyl-Phosphorsăure und Phosphoroxychlorid.

1. Wird Phospboroxychlorid und gewönliche Phosphorsäure in Mengen, welche der Gleichung:

$$
2 \mathrm{PO}_{4} \mathrm{I}_{3}+\mathrm{POCl}_{3}=3 \mathrm{PO}_{3} \mathrm{H}+3 \mathrm{ClH}
$$

entsprechen, zusammengebracht, so vermischen sie sich vollständig, ohne dass bei gewöhnlicher Temperattr eine Einwirkung zu bemerken wäre. Wird im Wasserbade erwärmt, so beginnt dieselbe und setzt sich bis zu Ende 
360 Geuther: Ueb. d. Einwirkg. der Phosphorchloride

fort unter Entwickelung von Chlorwasserstoff. Der zähe Rückstand in Wasser gelöst fällt Liweiss und wird durch Phosphorpentachlorid (siehe unten IV) zu Phosphoroxychlorid und Chlorwasserstoff, ist also Mouhydroxylphosphorsäure. ${ }^{1)}$

2. Wendet man weniger Oxychlorid an und $z$ war Mengen, welche der Gleichung:

$$
5 \mathrm{PO}_{4} \mathrm{H}_{3}+\mathrm{POCl}_{3}=3 \mathrm{P}_{2} \mathrm{O}_{7} \mathrm{H}_{4}+3 \mathrm{ClH}
$$

entsprechen, so verläuft die Reaction ähnlich, aber der dicke Rückstand besteht nicht aus einem Gemenge von Monhydroxyl- und Trihydroxyl-Phosphorsäure, sondern aus Pyrophosphorsäure, denn seine wässerige Lösung fällt Eiweiss nicht und giebt mit Argentinitrat auf vorsichtigen Zusatz von Ammoniak eine weisse Fällung.

$\mathrm{Da}$ sich die obige Gleichung aus den 2 Gleichungen: $2 \mathrm{PO}_{4} \mathrm{H}_{3}+\mathrm{POCl}_{3}=3 \mathrm{PO}_{3} \mathrm{H}+3 \mathrm{ClH}$ $8 \mathrm{PO}_{4} \mathrm{H}_{3}+3 \mathrm{PO}_{3} \mathrm{H}=3 \mathrm{P}_{2} \mathrm{O}_{7} \mathrm{H}_{4}$

zusammensetzen lässt, und also die Pyrophosphorsäure ihre Entstehung der Einwirkung von Monhydroxylphosphorsäure auf 'Trihydroxylphosphorsäure verdanken kann, so wurden diese beiden Säuren zu gleichen Mischungsgewichten zusammengebracht und im Wasserbade erwärmt. Nach längerer Einwirkung wird das Gemisch homogen und giebt nun mit Eiweiss keine, mit Silbersalzen aber eine weisse Fällung. Es entsteht also bei der Einwirkung von Monhydroxylphosphorsäure auf Tribydroxylphosphorsäure in der That Pyrophosphorsäure.

\section{Tribydroxyl-Phosphorsäure und Phosphor- pentachlorid.}

In der Voraussetzung, je nach der Menge des angewandten Chlorids würde die Einwirkung nach folgenden beiden Gleichnngen verlaufen:

1) Dieser und die folgenden Versuche, mit Ausnahmo der auf die untexphosphorige Süure bezüglichen, wurden im Winter 1871/72 ausgefuiturt. Seitdem hat auch Schiff (Ann. Chem. Pharn. 16B, 229) die Zersetzung dor dreibas. Phosphorsäure durch Phosphoroxychlorid io gleicher Weise beobachtet, 


$$
\begin{aligned}
3 \mathrm{PO}_{4} \mathrm{H}_{3}+\mathrm{PCl}_{\epsilon} & =4 \mathrm{PO}_{3} \mathrm{H}+5 \mathrm{ClH} \\
\mathrm{PO}_{4} \mathrm{H}_{3}+3 \mathrm{PCl}_{6} & =4 \mathrm{POCl}_{3}+3 \mathrm{ClH}
\end{aligned}
$$

und in der Voraussetzung, es würdo, da sich die letztere Gleichung aus den folgenden beiden zusammensetzt:

$$
\begin{aligned}
2 \mathrm{PO}_{4} \mathrm{H}_{3}+\mathrm{PCl}_{5} & =4 \mathrm{PO}_{3} \mathrm{H}+5 \mathrm{CLH} \\
\mathrm{PO}_{3} \mathrm{H}+2 \mathrm{PCl}_{6} & =3 \mathrm{POCl}_{2}+\mathrm{ClH}
\end{aligned}
$$

die erstere Gleichung stets zunächst realisirt werden, wurde Phosphorsäure und Phosphorpentachlorid in solchen der ersten Gleichung entsprechenden Mengen zusammengebracht. Es hatte sofort lebhafte Einwirkung ohne bedeutende Erwärmung unter Entwicklung von Chlorwasserstoff statt. Als das Phosphorpentachlorid verschwunden war, wurde das gleichförmig flüssige Gemisch auf dem Wasserbade erhitzt. Dabei traten ganz dieselben Erscheinungen ein, wie bei der Einwirkung von Phosphoroxychlorid auf Trihydroxyl-Phosphorsäure: es begann erneute Chlorwasserstoffentwicklung, bis zuletzt ein Rückstand vun Motaphosphorsäure blieb.

Daraus folgt also, dass bei gew öhnlicher Temperatur das Phosphorpentachlorid anf dreibas. Phosphorsänre nicht nach der oben angeführten ersten Weise, sondern stets sofort nach der zweiten Gleichung wirkt, d. h. unter Bildung von Phosphoroxychlorid, auch wenn TribydroxylPhosphorsäure im Ueberschuss vorhanden ist, and dass diese erst in der Würme durch das gebildete Phosphoroxychlorid weiter in Moahydroxylphosphorsäure nach der oben unter I. 1. angegebenen Art verwandelt wird.

\section{Trihydroxyl-Phosphorsäure und Phosphor- cblorür.}

Es wurden der Gieichung:

$$
3 \mathrm{PO}_{4} \mathrm{H}_{3}+\mathrm{PCl}_{3}=8 \mathrm{PO}_{3} \mathrm{H}+\mathrm{P}(\mathrm{OH})_{3}+3 \mathrm{ClH}
$$
entsprechende Mengen angewandt. Bei gewöhnlicher Temperatur findet keine Einwirkung statt, das Phosphorchlorür schwimmt unvermischt auf der Phosphorsäure. In der Wärme des Wasserbades beginnt eine gelinde Einwirkung, die sich durch Abscheidung von gelbem Phosphor bemerklich macht. Alluählich vermehrt sich dieser unter 
362 Geuther: Ueb. d. Einwirkg. der Phosphorehloride

Versehwinden des Phosphorchlorürs. Der verbleibende Rückstand giebt die Reactionen auf Py rophosphorsäure: Silbersalze werden weiss, aber Eiweiss wird nicht gefällt. Aus diesem Verhalten geht offenbar hervor, dass die Umsetzung nach der obigen Gleichung vor sich geht, aber es wird einestheils, wie bekannt, allmählich die gebildete phosphorige Săare darch das noch unveränderte Phosphorchlorïr in Phosphor, Chlorwasserstoff und gewöhnliche Phosphorsäure zerlegt; anderntheils wird die gebildete Monhydroxylphosphorsäure mit unveränderter Trihydroxylphosphorsäure zu Pyrophosphorsäure, wie unter I. 2. angeführt ist.

\section{Monhydroxylphosphorsäure und Phosphor- pentachlorid.}

Die beiden Verbindungen wirken bei gewöhnlicher Temperatur so gut wie nicht aufeinander ein, beim Erwärmen im Wasserbade aber beginnt starke Chlorwasserstoffentwickelung unter Verffüssigung der Masse. Wendet man genügend Chlorid an, so wird allmählich alles flüssig und in Phosphoroxychlorid verwandelt nach der Gleichung:

$$
\mathrm{PO}_{3} \mathrm{H}+2 \mathrm{PCl}_{5}=3 \mathrm{POCl}_{3}+\mathrm{Cl} \mathrm{H} \text {. }
$$

Als nur halb so viel Chlorid einwirken gelassen wurde, in der Hoffnung, neben gewöhnlichem Oxychlorid das Chlorid der Monhydroxylsäure zu erhalten, nach der Gleichung:

$$
\mathrm{PO}_{3} \mathrm{H}+\mathrm{PCl}_{5}=\mathrm{PO}_{2} \mathrm{Cl}+\mathrm{POCl}_{3}+\mathrm{ClH}
$$

fand doch die Einwirkung nur nach der ersten Gleichung statt, indem die Hälfte der Monhydroxylphosphorsäure unverändert blieb. Dies auffallende Resultat ist nur zu erklären entweder dadurch, dass das Metaphospborsäurechlorid sich sofort nach seinem Entstehen mit der vorhandenen Säure weiter in Phosphorsäureankydrid und Chlorwasserstuff nach der Gleichung: $\mathrm{PO}_{2} \mathrm{Cl}+\mathrm{PO}_{3} \mathrm{H}=\mathrm{P}_{2} \mathrm{O}_{5}+\mathrm{ClH}$ unsetzt und gewiönnliches Oxychlorid erzeugt, oder aber dadureh, dass das Metaphosphoreäurechlorid vom Phosphorpentachlorid selbst wieder angegriffen und $1 \mathrm{Mgt}$. 
Sauerstoft des Ersteren gegen die 2 Mgte. locker gebundenes Chlor des Letzteren unter Bildung von gewöhnlichem Oxychlorid nach der Gleichung: $\mathrm{PO}_{2} \mathrm{Cl}+\mathrm{PCl}_{5}=2 \mathrm{POCl}_{3}$ ausgewechselt wird, wie es ähnlich bei der Einwirkuug von $\mathrm{PCl}_{5}$ auf $\mathrm{Pyr}_{\mathrm{s}}$ phosphorsäurechlorid ja geschieht ${ }^{1}$ ).

Monhydroxyl-Phosphorsäure wird von Phosphoroxychlorid und Phosphorehlor ür bei der Siedetemperatur der Chloride nicht verändert.

V. Pyrophosphorsäure und Phosphoroxychlorid.

In der Kälte findet zwischen den beiden Verbindungen keine Einwirkung statt, das Oxychlorid überschichtot die Säure, ohne sich damit zu vermischen. In der Wasserbadwärme beginnt die Umsetzung unter sehäumender Entwicksung von Salzsäure und unter Trübwerden des Oxychlorids, bedingt durch die Abscheidung von Monhydroxylphosphorsäure, vollendet sich aber kaum, da selbst noch nach sehr langer Einwirkung Oxychlorid übrig ist und erst bei stärkerer Erwärmung von Neuem unter starkem Schäumen der immer zäher werdenden Masse einzuwirken beginnt. Die Gleichung, nach welcher die Umsetzung erfolgt, ist:

$$
2 \mathrm{P}_{2} \mathrm{O}_{7} \mathrm{H}_{4}+\mathrm{POCl}_{3}=5 \mathrm{PO}_{3} \mathrm{H}+3 \mathrm{ClII} \text {. }
$$

VI. Pyrophosphorsäure und Phosphorpentachlorid.

In der Kälte findet nur geringe Einwirkung statt, im Wasserbade wird sie lebhaft und vollendet sich unter Bildung von Phosphoroxychlorid und Chlorwasserstoff; wenn geu ügend Phosphorpentachlorid angewandt wurde, nach der Gleichung:

$$
\mathrm{P}_{2} \mathrm{O}_{7} \mathrm{H}_{4}+5 \mathrm{PCl}_{5}=7 \mathrm{POCl}_{3}+4 \mathrm{ClH} \text {. }
$$

Wird aher weniger Phosphorpentachlorid angewandt, so bleibt Monhydroxylphosphorsäure übrig, d. h. dann findet der Hergang zunächst nach der Gleichung statt:

$$
\mathrm{P}_{2} \mathrm{O}_{7} \mathrm{H}_{4}+\mathrm{PCl}_{6}=2 \mathrm{PO}_{3} \mathrm{H}+\mathrm{FOCl}_{8}+2 \mathrm{ClH} \text {. }
$$

1) Vergl. Jenser Zeitachrift 7, 105. 
364 Geuther: Ueb. d. Einwirkg. der Phosphorchloride

In keinem Falle wird die Bildung des Pyrophosphorsäurechlorids beobachtet, was möglicher Weise daher kommen kann, dass dasselbe, wie bekannt, sich mit Phosphorpentachlorid bei Wasserbadhitze in gewöhnliches Oxy. ehlorid verwandelt.

VII. Pyrophosphorsäure and Phosphorchlorür.

In der Kälte schwimmt das Phosphorehlorür unvermischt auf der Pyrophosphorsäure und ist ohne Einwirkung, auch selbst nach längerem Erwärmen auf dem Wasserbade bemerkt man kaum eine Veränderung, lässt man aber ein kleines Flämmchen direct so wirken, dass das Phosphorchlorür vom umgekehrten Kühler lebhaft zurückfliesst, so tritt die Entwicklung von Chlorwasserstoff auf und man bemerkt bald ein Trübwerden der vorher klar aussehenden Säure, genau so, wie wenn sich Monhydroxylphosphorsäure abscheidet; daneben findet gleichzeitig die Bildung von rothem Phosphor statt, wie bei der Zersetzung von phosphoriger Säure durch Phosphorchlorür. Es setzt sich dies fort und wenn man Mengen nach der Gleichung:

$$
3 \mathrm{P}_{2} \mathrm{O}_{7} \mathrm{H}_{4}+\mathrm{PCl}_{3}=6 \mathrm{PO}_{3} \mathrm{H}+\mathrm{P}(\mathrm{OH})_{3}+3 \mathrm{ClH}
$$

anwendet, so verschwindet das Phosphorchlorür vollständig und nun enthält der zähe rothe Rückstand Mon hydroxylphosphorsäure, denn seine filtrirte Lösung fällt Eiweiss. Es unterliegt somit keinem Zweifel, dass die eben angeführte Gleichung die wahre Umsetzungsgleichung darstellt, dass aber die entstandene phosphorige Säure weiter durch das Phosphorchlorür unter Phosphorabscheidung wio bekannt verändert wird. Das schliessliche Resultat der Einwirkung ergiebt sich als Sumane der 3 folgenden Reactionen:

$$
\begin{aligned}
12 \mathrm{P}_{2} \mathrm{O}_{7} \mathrm{H}_{4}+4 \mathrm{PCl}_{3} & =24 \mathrm{PO}_{9} \mathrm{H}+4 \mathrm{P}(\mathrm{OH})_{9}+12 \mathrm{ClH} \\
4 \mathrm{P}_{0}\left(\mathrm{OH}_{3}+\mathrm{PCl}_{3}\right. & =3 \mathrm{PO}_{4} \mathrm{H}_{3}+2 \mathrm{P}+3 \mathrm{ClH} \\
3 \mathrm{PO}_{4} \mathrm{H}_{3}+3 \mathrm{PO}_{3} \mathrm{H} & =3 \mathrm{P}_{2} \mathrm{O}_{7} \mathrm{H}_{4} \\
\hline 8 \mathrm{P}_{2} \mathrm{O}_{7} \mathrm{H}_{4}+5 \mathrm{PCl}_{3} & =21 \mathrm{PO}_{3} \mathrm{H}+2 \mathrm{P}+15 \mathrm{ClH}
\end{aligned}
$$


VIII. Phosphorige Säuro und Phosphoroxychlorid.

In der Erwartung, dass die Einwirkung des Phosphoroxychlorids auf die phosphorige Süure nach der Gleichung:

$3 \mathrm{POCl}_{3}+2 \mathrm{P}(\mathrm{OH})_{3}=3 \mathrm{PO}_{3} \mathrm{H}+2 \mathrm{PCl}_{3}+3 \mathrm{ClH}$

welche sich aus den beiden Gleichungen:

$$
\begin{aligned}
& \mathrm{POCl}_{3}+\mathrm{P}(\mathrm{OH})_{3}=\mathrm{PO}_{4} \mathrm{H}_{3}+\mathrm{PCl}_{3} \\
& \mathrm{POCl}_{3}+2 \mathrm{PO}_{4} \mathrm{H}_{3}=3 \mathrm{PO}_{3} \mathrm{H}+3 \mathrm{CHH}
\end{aligned}
$$

zusammensetzt, verlaufen würde, wurden die dieser Gleichung entsprechenden Mengen beider Verbindungen auf einander einwirken gelassen. Es findet gleichförmige Mischung unter geringer Erwärmnng und Entwicklung von viel Chlorwasserstoff statt. Die Reaction wurde sich im Wasserbade vollenden gelassen und darin so lange erhitzt, als noch Entwicklung von Chlorwasserstoff zu bemerken war. Es hatten sich nun zwei Schichten gebildet, eine dicke zähe untere und eine leicht bewegliche obere. Letztere wurde nach dem Erkalten abgegossen und destillirt; sie erwies sich als reines Phosphorchlorür. Die zähe untere Schicht hatte das Aussehen von Monhydroxylphosphorsäure; sie löste sich in Wasser unter geringer Erwärmung zu einer Flüssigkeit, welche Bariumchlorid und auch nach Zasatz von Natriumacetat Eiweisslösung fallte. Dass sie in der That Monhydroxylphosphorsäure war, wurde durch Zugabe der berechneten Menge von Phosphorsuperchlorid und Erwärmen im Wasserbade nachgewiesen, wobei sie unter Entwicklung ron Chlorwasserstoff und Bildnng von Phosphorchlorid verschwand

Die Thatsache, dass die phosphorige Säure mit Hülfe von Phosphoroxychlorid so leicht wieder in Phosphorchlorür, aus dem sie gabildet wird, zurückverwandelt werden kaun, hat eine wesentliche Bedeutang für die Constitution derselben. Sie zeigt unwiderruflich, dass Phosphorchlorür und phosphorige Säure darchaus in Verhältniss engster Zusammengehörigkeit stehen, dass eben das Erstere das zur Letzteren gehörige Chlorür ist, oder mit anderen Worten, dass die phosphorige 
366 Geuther: Ueb. d. Finwirkg. der Phosphorchloride

Sänreso gut wie das Phosphorchlorür trivalenten Phosphor enthält und die Formel der Ersteren also nieht $\stackrel{\mathrm{V}}{\mathrm{P}} \mathrm{HO}(\mathrm{OH})_{2}$, sondern $\stackrel{\mathrm{III}}{\mathrm{P}}(\mathrm{OH})_{3}$ ist.

\section{Phosphorige Säure und Phosphorpenta- chlorid.}

Das Phosphorchlorid wirkt lebhaft und unter starker Salzsäureentwicklung aber ohne bedeutende Erwärnung auf die phosphorige Säure ein. Wendet man auf $1 \mathrm{Mgt}$. der Säure zunächst nur $1 \mathrm{Mgt}$. des Chlorids an, so verflüssigt sich das luetztere leicht zu einem anfangs homogenen klaren Product. Später tritt ein Opalisiren der Flüssigkeit ein und es sondert sich eine dickere sehwerere Schicht ab. Wird die obere Schicht davon abgegossen und fiir sich nntersucht, so findet man leicht, dass sie aus Phosphorchlorïr und Phosphoroxychlorid besteht. Die untere dicke Flüssigkeit aber ist unveränderte phosphorige Säure völlig frei von Monhydroxylphosphorsäure. Lässt man sie auf ein zweites Mischungsgewicht Phosphorpentachlorid einwirken, so geschieht dasselbe. Die gavze Menge der phosphorigen Säure verschwindet erst, wenn auf $1 \mathrm{Mgt}$. derselben $3 \mathrm{Mgte}$. Pentachlorid angewandt werden nach der Gleichung:

$$
\mathrm{P}(\mathrm{OH})_{3}+3 \mathrm{PCl}_{5}=\mathrm{PCl}_{8}+3 \mathrm{POCl}_{3}+3 \mathrm{Cl} \mathrm{H} \text {. }
$$

X. Unterphosphorige Sänre und Phosphorchlotür.

Die zn diesem und den folgenden Versuchen verwandte unterphosphorige Säure war aus dem Barimsalz gewonnen worden, welches mit der genauen Menge von verdünnter Schwefelsäure ${ }^{\natural}$ in der Kälte zerlegt worden war. Die

1) Hat man eine Spur Schwetelsäure zuviel zugefügt, d. li. so wenig, dass sich in sehr verdtinnter Lösung dieselhe nioht mehr erkennen lässt, so kommt dieselbe während des Eindamptens als Sohwefel zum Vorschein, oder auoh noch beim Stehen über Schwefelsaure, woboi dio unterphosphorige Sänre allmähljch eine immer intensiver werdende tief indigoblaue Farbe animmt, welche mit der Zeit unter Schwefelabscheidung und Bildung von Sohtrefelwasserstoff immer heller wird and allmähich iu einen ganz schwach bräunliohen Ton ibergeht. 
filtrirte dünne Säure wurde erst in müssiger Wärme, zuletzt auf dem Wasserbade so lange concentrirt, bis der Geruch nach Phosphorwasserstoff aufzutreten begann und dann längero Zeit über Schwefelsäure gestellt. Sie war farblos, von öliger Consistenz, besass das spec. Gewicht 1,49 bei $+10^{\circ}$ und war ganz rein, wie die folgende Analyse zeigt.

0,4485 Grm. unterphosphorige Säure lieferten nach der Oxydation mittelst Salpetersäure und Königswasser 1) 0,7541 Grm. Magne. siumpyrophosphat, entspr. 0,2106 Grm. $=46,93$ p.C. Phosphor. Für die unterphosphorige Säure berechnen sich: 46,97 p.C Phosphor.

Das Phosphorchlorür wirkt sehr lebhaft unter starker Wärmeentwicklung auf die unterphosphorige Säure ein, weshalb man am Besten dasselbe tropfenweise zu der durch kaltes Wasser gekühlten Säure fliessen lässt. Das zufliessende Chlorür bewirkt sofort Umsetzung unter Entwicklung von Chlorwasserstoff und Bildnng von Phosphor, der anfangs als gelbe Haut, später als orangefarbene bis orangerothe Masse sich abscheidet. Zur

1) Oxydirt man die unterphosphorige Säure mittelst Salpetersäure, so tritt beim Erwärmen, auch wenn nur mässig starke Säure angewandt worden war, bald die Entwicklung rother Dämpfe ein. Bei weiterem Eindampfen hört dieselbe wieder auf und die wegdampfendo Salpetersäure ist scheinbar ohne weitere Einwirkung, bis dann bei genügender Concentration mit einem Male wieder eine reichliche und länger andauernde nene Fntwicklung rother Dämpfe beginnt. Die zuerst eintretende Entwicklung rother Dämpfe rührt von der Oxydation der unterphosphorigen Säure hauptsächlich nur zu phosphoriger Säure und die zu zweit eintretende ron der Oxydation der phosphorigen Sz̈ure zu Phosphorsäure her. Es ist lange schon bekannt, dass die Oxydation der phosphorigen Säure zu Phosphorsäure durch Salpetersäure nur sehr schwierig vollständig erreicht wird und dass ein wiederholtes starkes Eindampfen mit der letzteren Säure Noth thut. Ich habe mich ebenfalls hei Gelegenheit der obigen Analyse hiervon zu überzeugen Gelsgenheit gehabt. H. Rose bat deswegen vorgeschlagen, mit Salzsäure und Kalinmeblorat die Oxydation zu bewirken, ich habe aber niolit tindesi können, dass sie dadureh rascher und vollatändiger von statten goht. denn ein grosser Theil des Chlors entweicht stets obne oxydirend zu wirken. Vollständig und raseh erreicht wan die Oxydation indess, wem man nach der Oxydation mit Salpetershiure noch starkes Königswasser zufügt und eindampft, bis keine rothen Dumpfe mehr kommen. 
Vollendung der Einwirkung wurde schliesslich noeb am aufgerichteten Kihhler im Waseerbade erwärmt und zuletzt das überschüssigg gebliebene Chlorür abdestillirt. Der rothe zähe Rückstand wurde nun mit viel Wasser übergossen und verschlossen stehen gelassen. Fitwa $4 / 5$ desselben gehen in Lösung als phosphorige Säare und TrihyAroxylphosphorsäure, während $1 / 5$ als orangerother Phosphorl) übrig bleibt. Von der ersteren Säure ist am meisten, Monhydroxylphosphorsäure ist gar nicht vorhanden.

Die abgeschiedene Phosphormenge sowohl als die verbraucht werdende Menge von Phosphorehlorür zeigen, dass der Hergang bei der Umsetzang vorzüglich verläuft nach der Gleichung:

$3 \mathrm{PH}(\mathrm{OH})_{2}+\mathrm{PCl}_{3}=2 \mathrm{P}(\mathrm{OH})_{3}+2 \mathrm{P}+3 \mathrm{ClH}$.

Die mit entstandene Trihydroxylsäure verdankt ihre Entstehung einer nebenher gehenden Einwirkung des Phosphorchloriurs auf die phosphorige Säure nach bekannter Art und Weise. Die unterphosphorige Säure benimmt sich also in dieser Reaction wie ein Gemisch von Phosphorwasserstoff und phosphoriger Säure:

$$
3 \mathrm{PH}(\mathrm{OH})_{2}=\mathrm{PH}_{3}+2 \mathrm{P}(\mathrm{OH})_{3} \text {. }
$$

Dor Phosphorwasserstoff setzt sich dann, wie bekannt, mit dem Phosphorchlorir um in rothen Phosphor und Chlorwasserstoff, während die phosphorige Säure übrig bleibt.

Es will mir schoinen, als ob auch dies Verhalten dafür spräche, dass in der unterphosphorigen Säure trivalenter und nicht pentavalenter Phosphor enthalten, ihre Formel

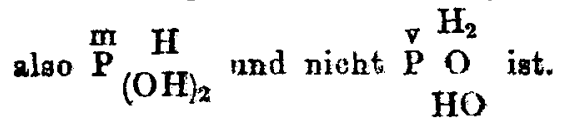

;) Derselbe mit heissem Wasser gewaschen, möglichst rasch durch Fliesepapier rom anhängenden $W$ asser befreit und im leeran Raum über Sohwefelshure getrocknet, ergab bei der Analyse 96-96,2 p.C. Phosphor. Die fehlenden 4 p.C. rühren jedenfalls von noob beigemeng. ten Säuren de Phosphors, hauptsächlich phoøphoriger Säure her, denn baim Brhitzen im Röhrohen entsteht neben viel sublimirenden Phosphor etwas entzändliches Phosphorwasserstoffgas und etwas Phospborsăure. 
Ein Chlorid der unterphosphorigen Säure dessen Bildung auf diese Weise hätte möglich sein können, entsteht also nicht, denn auch die kleinste Menge von Phosphorchlorür bewirkt sofort Abscheidung von Phosphor. Es geht daraus hervor, dass weder die Verbindung $\mathrm{PHCl}_{2}$ noch das Anhydrid der unterphosphorigen Säure $\mathrm{H}$

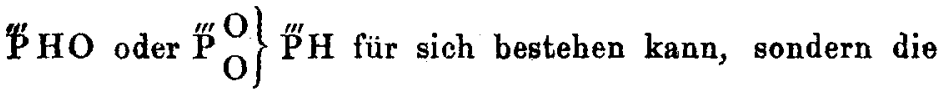
erstere nach der Gleichung: $3 \mathrm{PHCl}_{2}=\mathrm{PCl}_{3}+2 \mathrm{P}+3 \mathrm{ClH}$, der letztere aber nach der Gleichung: $3 \mathrm{PHO}=\mathrm{P}(\mathrm{OH})_{3}+2 \mathrm{P}$ zerfallen muss.

XI. Unterphosphorige Säure und Phosphoroxychlorid.

Die Einwirkung des Phosphoroxychlorids auf die unterphosphorige Süure ist scheinbar noch lebhafter als die des Phosphorchlorïrs und äusserlich von ganz ähnlichem Verlauf. Jeder Tropfen wirkt wie dort unter Phosphorabscheidung und Salzsäureentwicklang ein. Man verfährt deshalb auch hier wie dort. Wird nach vollendeter Einwirkung im Wasserbade mit aufsteigendem Kühler erhitzt, wobei eine erneute lebhaftere Entwicklung von Chlorwasserstoff auftritt, so bemerkt man bald, dass eine unter $100^{\circ}$ siedende Verbindung gebildet ist, indem Flüssigkeit bis in den Kühler destillirt und von da wieder zurüekfliesst. Dieselbe kann aus dem Wasserbade vom Rückstand und vom etwa überschüssig zugesetzten Oxychlorid abdestillirt werden und erweist sich bei erneuter Rectifioation als Phosphorchlorür. Der Rüekstand selbst besteht hauptsächlich aus Monhydroxylphosphorsäure. Der gebildeten Menge ausgeschiedenen Phosphors nach verläuft die Einwirkung nach den 2 Gleichungen:

$$
\begin{aligned}
6 \mathrm{PH}(\mathrm{OH})_{2}+3 \mathrm{POCl}_{3} & \left.=3 \mathrm{PO}_{3} \mathrm{H}+2 \mathrm{P} \mathrm{OH}\right)_{3}+4 \mathrm{P}+9 \mathrm{ClH} \\
2 \mathrm{P}(\mathrm{OH})_{3}+3 \mathrm{POCl}_{3} & =3 \mathrm{PO}_{3} \mathrm{H}+2 \mathrm{PCl}_{3}+3 \mathrm{CHH} \\
2\left[3 \mathrm{PH}(\mathrm{OH})_{2}+3 \mathrm{POCl}_{3}\right. & \left.=3 \mathrm{PO}_{3} \mathrm{H}+\mathrm{PCl}_{3}+2 \mathrm{P}+6 \mathrm{ClH}\right]
\end{aligned}
$$

Dass sie sich nach der ersteren Gleichung zunächst realisirt, wird rlurch die oben erwähnte erneute EntwiakJouru. f. prakt. Chemle [2] Bd, 8. 
lung von Chlorwasserstoffgas, welche bei der Einwirkung des Wasserbades statt hat, sehr wahrscheinlich gemacht. Der Rückstand enthïlt auch neben Metaphosphorsäure eine das Quecksilberchlorid redticirende Süure, indess kann daraus nicht auf die Anwesenheit von phosphoriger Säure geschlossen werden, da die sich bildende zäho Metaphosphorsäure leicht etwas unverändert gebliebene unterphosphorige Säure oder etwas Phosphorchlorür einschliessen kann.

Die erstere Gleichung lässt sich nun aber weiter als die Summe folgender einfachen and sehr verständlichen Gleichungen auffassen:

$$
\begin{gathered}
6 \mathrm{PH}(\mathrm{OH})_{2}=2 \mathrm{PH}_{3}+4 \mathrm{P}(\mathrm{OH})_{3} \\
4 \mathrm{P}(\mathrm{OH})_{3}+3 \mathrm{POCl}_{3}=3 \mathrm{PO}_{3} \mathrm{H}+2 \mathrm{PCl}_{3}+8 \mathrm{ClH}+2 \mathrm{P}(\mathrm{OH})_{3} \\
2 \mathrm{PH}_{3}+2 \mathrm{PCl}_{3}=4 \mathrm{P}+6 \mathrm{CH}
\end{gathered}
$$

d. b. die unterphosphorige Säure verhält sich dem Phosphoroxychlorid gegenüber wie ein Gemisch von Phosphorwasserstoff und phosphoriger Säure, also ebenso, wie sie es dem Phosphorchloritr gegevuiber that ${ }^{2}$ ). So findet die dort aus diesem Verhalten hergeleitete An. sicht über die Constitution der Säure also auch von hier aus theue Unterstilizung.

\section{Unterphosphorige Săure und Phosphor- pentachlorid.}

Nach der Kenntniss der Einwirkung des Phosphoroxychlorids auf die unterphosphorige Säure war es nicht schwer, die Gleichung für die Endreaction aufzustellen, welche sich als die Summe der vier folgenden ergielt:

1) Auch beim Erhitzen für sich verblt sioh die unterphosphorige säure so. Es ist sohon oben erwähnt, dass oie bem reits bei $100^{\circ}$ anfangt Phosphorwasserstoff zu entwickeln. Dasselbo geschieht rasch unter betrïchtlichem Schänmen zwischen 110 and $115^{\circ}$. Der verbleibende Rückstand ist phosphorige Säure, denn er kann bis $250^{\circ}$ obne Veränderung erbitzt werden, während er tuber diese Temperatur hinaug erwärmt, ron Neuem Phosphorwasserstoff zu entwickeln beginnt nnd nun erst einen Riickstand von Phoophorsabre lïsst. 


$$
\begin{aligned}
3 \mathrm{PH}(\mathrm{OH})_{2}+6 \mathrm{PCl}_{5} & =6 \mathrm{POCl}_{3}+\mathrm{PCl}_{3}+2 \mathrm{P}+9 \mathrm{CHH} \\
6 \mathrm{PH}(\mathrm{OH})_{2}+6 \mathrm{POCl}_{3} & =6 \mathrm{PO}_{3} \mathrm{H}+2 \mathrm{PCl}_{3}+4 \mathrm{P}+12 \mathrm{CH} \\
6 \mathrm{PO}_{3} \mathrm{H}+12 \mathrm{PCl}_{5} & =18 \mathrm{POCl}_{3}+6 \mathrm{ClH} \\
6 \mathrm{P}+9 \mathrm{PCl}_{5} & =15 \mathrm{PCl}_{3} \\
\hline 9\left[\mathrm{PH}(\mathrm{OH})_{2}+3 \mathrm{PCl}_{5}\right. & \left.=2 \mathrm{POCl}_{3}+2 \mathrm{PCl}_{3}+3 \mathrm{CH}\right] .
\end{aligned}
$$

Es war vielmehr durch den Versuch festzustellen, ob bei der Einwirkung des Phosphorpentachlorids sofort die Umsetzung nach der Endreaction verlaufen würde oder ob erst die Zwischenreactionen sich verwirklichten. An einer eintretenden Phosphorabscheidung war dies leicht zu erkennen.

Fügt man zu unterphosphoriger Süure, die durch kaltes Wasser gekuihlt ist, allmählich und in kleineren Portionen das nach obiger Endgleichung berechnete Phosphorpentachlorid, so findet lebhafte Einwirkung anter starker Chlorwasserstoffentwicklung und sofortiger Abscheidung von rothem Phosphor statt. Es setzt sich dies beim Zufügen neuer Mengen des Chlorids eine Zeit lang so fort, die Einwirkung wird allmählich schwächer und zuletzt so schwach, dass der noch verbliebene grosse Rest von Chlorid auf einmal zngegeben werden kann und zur weiteren Hinwirkung gelinde Würme des Wasserbades angewandt werden muss. Schliesslich ist alles verthüssigt und besteht aus einem Genenge von Phosphoroxychlorid und Phosphorchlorür.

Die Gleichungen, nach welchen die Einwirkung der Phosphorchloride auf die Phosphorsüuren zunächst verläuft, sind demnach die folgenden:

$$
\begin{aligned}
\mathrm{PO}_{3} \mathrm{H}+2 \mathrm{POl}_{5} & =3 \mathrm{POCl}_{3}+\mathrm{CHH} . \\
3 \mathrm{PO}_{4} \mathrm{H}_{3}+\mathrm{PCl}_{3} & =3 \mathrm{PO}_{3} \mathrm{H}+\mathrm{P}(\mathrm{OH})_{3}+3 \mathrm{CH} . \\
2 \mathrm{PO}_{4} \mathrm{H}_{3}+\mathrm{POCl}_{3} & =3 \mathrm{PO}_{3} \mathrm{H}+3 \mathrm{ClH} \\
\mathrm{PO}_{4} \mathrm{H}_{3}+3 \mathrm{PCl}_{5} & =4 \mathrm{POCl}_{3}+3 \mathrm{ClH} . \\
3 \mathrm{P}_{2} \mathrm{O}_{7} \mathrm{H}_{4}+\mathrm{PCl}_{3} & =6 \mathrm{PO}_{3} \mathrm{H}+\mathrm{P}(\mathrm{OH})_{3}+3 \mathrm{CHH} \\
2 \mathrm{P}_{2} \mathrm{O}_{7} \mathrm{H}_{4}+\mathrm{POCl}_{3} & =5 \mathrm{PO}_{3} \mathrm{H}+3 \mathrm{CHH} \\
\mathrm{P}_{2} \mathrm{O}_{7} \mathrm{H}_{4}+\mathrm{PCl}_{5} & =2 \mathrm{PO}_{3} \mathrm{H}+\mathrm{POCl}_{3}+2 \mathrm{CHH} .
\end{aligned}
$$


372 Geuther: Untersuchung über sauerstoffreiche

$$
\begin{aligned}
& 4 \mathrm{P}(\mathrm{OH})_{3}+\mathrm{PCl}_{3}=3 \mathrm{PO}_{4} \mathrm{H}_{3}+2 \mathrm{P}+3 \mathrm{ClH} \text {. } \\
& 4 \mathrm{P}(\mathrm{OH})_{3}+3 \mathrm{POCl}_{3}=3 \mathrm{PO}_{3} \mathrm{E}+2 \mathrm{PCl}_{3}+3 \mathrm{CHH} \text {. } \\
& \mathrm{P}(\mathrm{OH})_{3}+3 \mathrm{PCl}_{5}=3 \mathrm{POCl}_{3}+\mathrm{PCl}_{3}+8 \mathrm{CH} \text {. } \\
& \mathrm{BPH}(\mathrm{OH})_{2}+\mathrm{PCl}_{3}=2 \mathrm{P}(\mathrm{OH})_{3}+2 \mathrm{P}+3 \mathrm{ClH} \text {. } \\
& 6 \mathrm{PH}(\mathrm{OH})_{2}+3 \mathrm{POCl}_{3}=2 \mathrm{P}(\mathrm{OH})_{3}+4 \mathrm{P}+3 \mathrm{PO}_{8} \mathrm{H}+9 \mathrm{ClH} \text {. } \\
& 8 \mathrm{PH}(\mathrm{OH})_{2}+6 \mathrm{PCl}_{5}=\mathrm{PCl}_{3}+2 \mathrm{P}+6 \mathrm{POCl}_{3}+8 \mathrm{CH} \text {. }
\end{aligned}
$$

\section{Untersuchung über sauerstoffreiche Kohlen- stoffsäuren; \\ ron}

\section{A. $Q \operatorname{euth} \theta \mathbf{x}$.}

Vor nunmehr fünf Jahren wurde die erste Abhandlung über diesen Gegenstand veröffentlicht ${ }^{1}$ ). Es war dies die Abhandlung Riemann's über die Einwirkung der concentrirten Salzsäure auf Weinsäure und Traubensäure in höherer Temperatur. Damals habe ich erwähnt, dass auch Versuche in gleicher Richtung mit der Citronensäure unternommen worden seien, welche zur Kenntniss zweier neuer Säuren von der Zusammensetzung: $\mathrm{C}_{10} \mathrm{H}_{12} \mathrm{O}_{9}$ und $\mathrm{C}_{9} \mathrm{H}_{40} \mathrm{O}_{6}$ geführt hätten. Die nähere Untersuchung dieses Vorganges dureh Herrn Dr. O. Hergt, welche im Folgenden niedergelegt ist, zeigt, dass nur die letztere Säure als Zersetzungsproduet auftritt, die erstere dagegen sich als unreine und modificirte Citronensäure ergeben hat.

\section{Abhandiung.}

Ueber die Einwirkung von concentrirter Chlor. wasserstoffsäure auf Citronensäure in höheren Temperaturen; von Dr. Otto Hergt.

Je nach der Temperatur, bei welcher die Einwirkung der Salzzäure auf Citronensäure stattfindet, sind die Pro-

3) Jenaer Zeitschrift 4, 288. 